\title{
Evaluation of Muscle Cramp Associated with Liver Cirrhosis with a Focus on the Liver Function and Nutritional Status
}

\author{
Masaaki Shimada ${ }^{1}$, Noboru Hirashima ${ }^{1}$, Hiroaki Iwase ${ }^{1}$, Masashi Saito ${ }^{1}$, Hisashi Kondo ${ }^{1}$, \\ Noboru Urata ${ }^{1}$, Satoshi Unita ${ }^{1}$, Takashi Kondo ${ }^{1}$, Daiki Tanaka ${ }^{1}$, \\ Takuya Tsunekawa ${ }^{1}$ and Mitsuhiro Fujishiro ${ }^{2}$
}

\begin{abstract}
:
Objective We investigated the muscle cramp status of patients with liver cirrhosis by focusing on the degree of liver damage, skeletal muscle mass, and nutritional status.

Methods All enrolled patients completed a questionnaire about muscle cramps. The degree of liver damage was examined using the Child-Pugh classification and the albumin-bilirubin (ALBI) grade. The nutritional status and skeletal muscle mass were examined using the Controlling Nutritional Status (CONUT) method and the psoas muscle index (PMI).

Results Among the respondents, 55.7\% of the patients reported experiencing muscle cramps. An analysis of the two patient groups-those who experienced muscle cramps and those who did not-revealed significant differences in Child-Pugh classification (muscle cramp-positive vs. muscle cramp-negative: A/B/C, $54.1 \% /$ $32.4 \% / 13.5 \%$ vs. $90.0 \% / 10.0 \% / 0.0 \%$; $\mathrm{p}=0.004)$, ALBI grade $(1 / 2 / 3,20.5 \% / 71.8 \% / 7.7 \%$ vs. $54.8 \% / 38.7 \% /$ $6.5 \%$; $\mathrm{p}=0.011)$, modified ALBI grade $(1 / 2 \mathrm{a} / 2 \mathrm{~b} / 3,20.5 \% / 20.5 \% / 51.3 \% / 7.7 \%$ vs. $54.8 \% / 22.6 \% / 16.1 \% / 6.5 \%$; $\mathrm{p}=0.008$ ), CONUT score (normal $/ \mathrm{mild} /$ moderate $/$ severe, $25.6 \% / 28.2 \% / 41.0 \% / 5.1 \%$ vs. $22.6 \% / 61.3 \% / 12.9 \% /$ $3.2 \%$; $=0.024)$, and PMI $\left(3.85 \pm 1.13 \mathrm{~cm}^{2} / \mathrm{m}^{2}\right.$ vs. $\left.4.94 \pm 1.86 \mathrm{~cm}^{2} / \mathrm{m}^{2} ; \mathrm{p}=0.012\right)$.

Conclusion Our findings suggest that muscle cramps occur more frequently in patients with liver cirrhosis due to their decreased liver function and poorer nutritional status.
\end{abstract}

Key words: albumin-bilirubin grade, Controlling Nutritional Status, liver cirrhosis, muscle cramps, psoas muscle index, Child-Pugh classification

(Intern Med 60: 1343-1348, 2021)

(DOI: 10.2169/internalmedicine.6231-20)

\section{Introduction}

Liver cirrhosis is classified as asymptomatic compensated cirrhosis or decompensated cirrhosis with obvious symptoms. Patients with decompensated liver cirrhosis are reported to have a poor prognosis (1). Painful spasms of the lower-limb muscles are experienced by $20-88 \%$ of patients with liver cirrhosis (2); they also reduce patient quality of life (QOL) (3). The severity of liver cirrhosis and ascites is related to the onset of muscle cramps (4). However, the effect of degree of liver damage on muscle cramps remains unclear (5).

The Child-Pugh classification (6), which is used to evaluate degree of liver damage, classifies patients from class $\mathrm{A}$ to $\mathrm{C}$ based on five factors: encephalopathy, ascites, serum total bilirubin (T.Bil) levels, serum albumin (Alb) levels, and prothrombin time (PT). A worse Child-Pugh classification indicates a worse prognosis $(1,7)$. However, encephalopathy and ascites are subjectively measured, and if all five factors

${ }^{1}$ Department of Gastroenterology, National Hospital Organization, Nagoya Medical Center, Japan and ${ }^{2}$ Department of Gastroenterology \& Hepatology, Nagoya University Graduate School of Medicine, Japan

Received: September 3, 2020; Accepted: October 22, 2020; Advance Publication by J-STAGE: December 7, 2020

Correspondence to Dr. Masaaki Shimada, shimada.masaaki.za@mail.hosp.go.jp 


\begin{tabular}{||l||} 
Please mark X on the circle that best describes your answer. \\
1. Have you experienced muscle cramps or terrible leg cramps in the last three months? \\
OYes \\
2. If daily, indicate the number of cramps per day. Repeat this for the "weekly" and \\
"monthly" choices. \\
Oevery day. If daily number of cramps/day__ \\
Oevery week. If weekly number of cramps/week \\
Oevery month. If monthly number of cramps/month_ \\
3. How long do your cramps last? \\
4few seconds Ominutes Ohours \\
5. How painful are your muscle cramps? Mark the line below according to the severity. \\
No pain at all Onight Oboth day and night
\end{tabular}

Figure 1. The questionnaire used to gather the characteristics of muscle cramps.

are not available, then the degree of liver damage cannot be evaluated.

In contrast, determining the albumin-bilirubin (ALBI) grade is simple, objective, and useful, and only requires the measurement of the patient's serum Alb and T.Bil levels. The ALBI grade is classified into grades 1-3. Modified ALBI (mALBI) grade, a subdivision of ALBI grade 2 into grades $2 \mathrm{a}$ and $2 \mathrm{~b}$, is also used $(8,9)$. The ALBI grade is also useful for predicting the outcomes of surgery (10), radiofrequency ablation (11), transcatheter arterial chemoembolization (12), and molecular-targeted therapy (13).

The Controlling Nutritional Status (CONUT) method is used to calculate a score based on serum Alb levels, the total lymphocyte count (TLC), and total cholesterol (T-cho). The patient's nutritional status is then classified as follows: normal (0-1), mild disorder (2-4), moderate disorder (5-8), and advanced disorder (9-12) (14). Approximately 63\% of patients with liver cirrhosis are reported to be malnourished, and the CONUT method can be used to evaluate their nutritional status (15). Furthermore, this method demonstrates superior performance in simple nutritional evaluations performed during ordinary medical care (16) and is useful for predicting the long-term prognosis of patients with end-stage liver disease (17).
The concept of sarcopenia was first proposed by Rosenberg in 1989 (18). In 2010, sarcopenia was defined by the European working group as “.... a syndrome characterized by progressive and generalized loss of muscle mass and strength, which is associated with physical dysfunction, poor QOL, and risk of death" (19). Even in Japan, patients with chronic liver disease are aging, are frequently malnourished, and have various comorbid metabolic disorders; these conditions place the patients at high risk of developing sarcopenia. Thus, in 2016, the Japan Society of Hepatology released the first edition of the assessment criteria for sarcopenia in liver disease (20).

The frequency, degree, and pathophysiology of muscle cramps in patients with liver cirrhosis have not been extensively studied. In particular, detailed examinations based on patient ALBI grade, nutritional status, and skeletal muscle mass have not been performed. This study aimed to investigate muscle cramps in patients with liver cirrhosis by focusing on the degree of liver damage, skeletal muscle mass, and nutritional status.

\section{Materials and Methods}

In this exploratory study, we used a questionnaire (Fig. 1) 
Table 1. Baseline Patient Characteristics.

\begin{tabular}{lc}
\hline Age (years) & $69.8 \pm 11.2$ \\
Sex (male/female) & $48 / 22$ \\
BMI $\left(\mathrm{kg} / \mathrm{m}^{2}\right)$ & $23.8 \pm 3.8$ \\
Etiology of chronic liver disease & \\
$\quad$ Alcoholic/hepatitis B/hepatitis C/PBC/AIH/IPH/others & $20 / 10 / 22 / 5 / 1 / 1 / 11$ \\
HCC & 23 \\
Alb (g/dL) & $3.7 \pm 0.7$ \\
T.Bil (mg/dL) & $1.33 \pm 1.20$ \\
PT $(\%)$ & $83.7 \pm 18.2$ \\
Child-Pugh classification (A/B/C) & $47 / 15 / 5$ \\
Child-Pugh score & $6.2 \pm 1.6$ \\
ALBI grade $(1 / 2 / 3)$ & $25 / 40 / 5$ \\
mALBI grade $(1 / 2 \mathrm{a} / 2 \mathrm{~b} / 3)$ & $25 / 15 / 25 / 5$ \\
ALBI score & $-2.30 \pm 0.62$ \\
CONUT (normal/low/moderate/severe) & $17 / 30 / 20 / 3$ \\
CONUT score & $3.7 \pm 2.4$ \\
PMI (cm $\left./ \mathrm{m}^{2}\right)$ & $4.31 \pm 1.57$ \\
\hline
\end{tabular}

Data are expressed as number or mean \pm standard deviation.

BMI: body mass index, PBC: primary biliary cholangitis, AIH: autoimmune hepatitis, IPH: idiopathic portal hypertension, HCC: hepatocellular carcinoma, Alb: albumin, T.Bil: total bilirubin, PT: prothrombin time, ALBI grade: albumin-bilirubin grade, mALBI grade: modified ALBI grade, CONUT: Controlling Nutritional Status, PMI: psoas muscle index

to conduct a survey of muscle cramps among 70 consecutive patients with liver cirrhosis who visited the Department of Gastroenterology at Nagoya Medical Center between October 2018 and June 2019. Data regarding each patient's background, blood test results (peripheral blood counts, liver function tests, Alb levels, PT, T-cho levels), and medications taken [e.g., Shakuyaku-kanzo-to, carnitine, and branchedchain amino acids (BCAAs)] were obtained from the patients' medical records. The degree of liver damage was examined using the Child-Pugh classification and ALBI grade. The nutritional status and skeletal muscle mass were examined using the CONUT method and psoas muscle index (PMI) measurements, respectively. PMI was determined by tracing an outline of the bilateral psoas major muscles using an image unification system (SYNAPSE SCOPE V2 4.1; Fujifilm Corporation, Tokyo, Japan) at the level of the third lumbar vertebra on abdominal axial computed tomography (CT). The total area was then divided by the square of the patient's height.

\section{Statistical analyses}

All data are expressed as the mean \pm standard deviation. Intergroup differences were analyzed using the MannWhitney test and chi-squared test. $\mathrm{p}$ values of $<0.05$ were considered to indicate statistical significance.

This study was approved by the Ethics Review Board of Nagoya Medical Center.

\section{Results}

\section{Baseline patient characteristics}

This study included 48 men and 22 women with a mean age of $69.8 \pm 11.2$ years. The etiologies of liver cirrhosis included: alcoholic liver disease $(n=20)$, hepatitis B infection $(n=10)$, hepatitis $C$ infection $(n=22)$, primary biliary cholangitis $(n=5)$, autoimmune hepatitis $(n=1)$, idiopathic portal hypertension $(n=1)$, and other $(n=11)$. The Child-Pugh classifications were as follows: class $A, n=47$, class $B, n=15$, and class $\mathrm{C}, \mathrm{n}=5$; the mean Child-Pugh score was $6.2 \pm 1.6$ (all five factors were not available for three patients). The mALBI grades were as follows: grade $1, n=25$, grade $2, n=$ 40 (grade $2 \mathrm{a}, \mathrm{n}=15$; grade $2 \mathrm{~b}, \mathrm{n}=25$ ), and grade $3, \mathrm{n}=5$. The mean ALBI score was $-2.30 \pm 0.62$ (Table 1).

\section{Frequency of muscle cramps}

Muscle cramps occurring within 3 months before the survey were reported by $55.7 \%$ of the respondents. Muscle cramps were experienced daily, weekly, and monthly by $14.7 \%, 47.1 \%$, and $38.2 \%$ of the respondents, respectively. The duration spanned seconds in $23.5 \%$ of the respondents, minutes in $70.6 \%$, and hours in $5.9 \%$. Overall, $67.6 \%$ of the respondents noted experiencing cramps at night, while the remaining $23.5 \%$ reported experiencing cramps during the day and at night. Patients experienced varying degrees of pain, as demonstrated by the range in the pain visual analog scale results (Fig. 2). 
$\mathbf{A}$

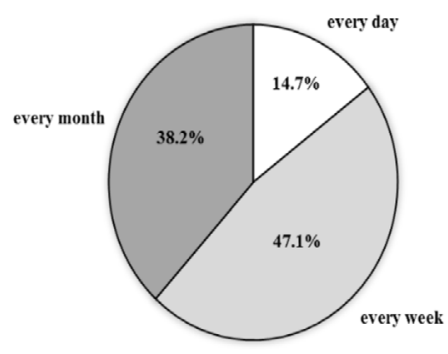

C

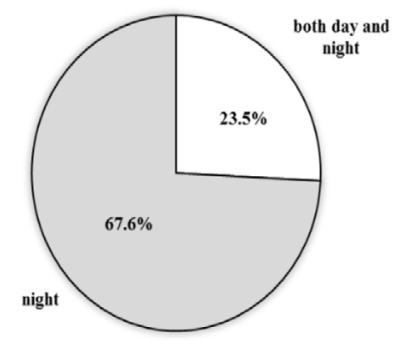

B

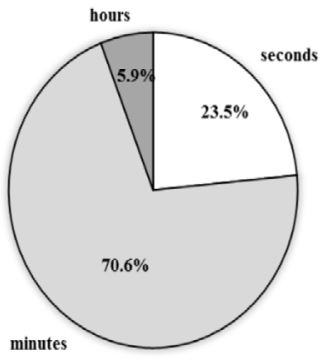

$\mathrm{n}=34$

D

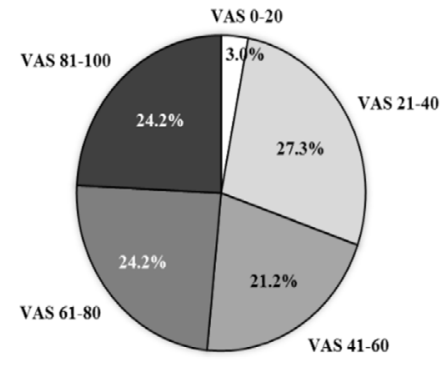

$\mathrm{n}=\mathbf{3 4}$

Figure 2. Frequency of muscle cramps (A), duration (B), time of onset (C), and severity of pain (D). VAS: visual analog scale

Table 2. Relationship between Muscle Cramps and the Child-Pugh Classification.

\begin{tabular}{lccc}
\hline & Presence of muscle cramps & Absence of muscle cramps & p value \\
\hline $\mathrm{n}$ & 37 & 30 & \\
Child-Pugh A/B/C $(\%)$ & $54.1 / 32.4 / 13.5$ & $90.0 / 10.0 / 0.0$ & 0.004 \\
Child-Pugh score & $6.7 \pm 1.7$ & $5.6 \pm 1.1$ & 0.002 \\
\hline
\end{tabular}

Table 3. Relationship between Muscle Cramps and ALBI Grade.

\begin{tabular}{lccc}
\hline & Presence of muscle cramps & Absence of muscle cramps & p value \\
\hline $\mathrm{n}$ & 39 & 31 & \\
ALBI grade $1 / 2 / 3(\%)$ & $20.5 / 71.8 / 7.7$ & $54.8 / 38.7 / 6.5$ & 0.011 \\
mALBI grade $1 / 2 \mathrm{a} / 2 \mathrm{~b} / 3(\%)$ & $20.5 / 20.5 / 51.3 / 7.7$ & $54.8 / 22.6 / 16.1 / 6.5$ & 0.008 \\
ALBI score & $-2.13 \pm 0.60$ & $-2.51 \pm 0.59$ & 0.003 \\
\hline
\end{tabular}

ALBI grade: albumin-bilirubin grade, mALBI grade: modified ALBI grade

Association of nutritional status, skeletal muscle mass, and degree of liver damage with muscle cramps

Significant differences were found between patients who experienced muscle cramps and those who did not in ChildPugh classification (muscle cramp-positive vs. muscle cramp negative: $\mathrm{A} / \mathrm{B} / \mathrm{C}: \quad 54.1 \% / 32.4 \% / 13.5 \%$ vs. $90.0 \% / 10.0 \% /$ $0.0 \% ; \mathrm{p}=0.004)$, ALBI grade (1/2/3: $20.5 \% / 71.8 \% / 7.7 \%$ vs. $54.8 \% / 38.7 \% / 6.5 \% ; \mathrm{p}=0.011)$, mALBI grade $(1 / 2 \mathrm{a} / 2 \mathrm{~b} / 3$ : $20.5 \% / 20.5 \% / 51.3 \% / 7.7 \%$ vs. $54.8 \% / 22.6 \% / 16.1 \% / 6.5 \%$; $=$ 0.008 ), CONUT classification (normal/mild/moderate/severe: $25.6 \% / 28.2 \% / 41.0 \% / 5.1 \%$ vs. $22.6 \% / 61.3 \% / 12.9 \% / 3.2 \%$; $=$ $0.024)$, and PMI $\left(\mathrm{cm}^{2} / \mathrm{m}^{2}: 3.85 \pm 1.13\right.$ vs. $\left.4.94 \pm 1.86 ; \mathrm{p}=0.012\right)$
(Table 2-4). A multivariate analysis with logistic regression revealed that the presence of muscle cramps was not significantly associated with Child-Pugh score, ALBI score, CONUT score, or PMI.

\section{Treatment for muscle cramps}

Thirty-two patients received treatment for muscle cramps with the following medications: BCAAs $(n=21)$, Shakuyakukanzo-to $(n=7)$, carnitine $(n=2)$, BCAAs + carnitine $(n=1)$, and BCAAs + Shakuyaku-kanzo-to $(n=1)$. Thirty-eight patients did not use drugs for muscle cramps. The muscle cramp frequency was significantly associated with the ALBI/ mALBI grade and (ALBI grade 1/2/3: 22.2\%/57.9\%/ $100.0 \%$; $\mathrm{p}=0.044$, mALBI grade $1 / 2 \mathrm{a} / 2 \mathrm{~b} / 3: 22.2 \%, 37.5 \%$, 
Table 4. Relationship between Muscle Cramps and Nutritional Status.

\begin{tabular}{lccc}
\hline & Presence of muscle cramps & Absence of muscle cramps & p value \\
\hline $\mathrm{n}$ & 39 & 31 & \\
CONUT normal/low/moderate/severe $(\%)$ & $25.6 / 28.2 / 41.0 / 5.1$ & $22.6 / 61.3 / 12.9 / 3.2$ & 0.024 \\
$\mathrm{PMI}\left(\mathrm{cm}^{2} / \mathrm{m}^{2}\right)$ & $3.85 \pm 1.13$ & $4.94 \pm 1.86$ & 0.012 \\
\hline
\end{tabular}

CONUT: Controlling Nutritional Status, PMI: psoas muscle index

$42.7 \%$, and $100.0 \%$; $\mathrm{p}=0.035)$ but not the Child-Pugh classification (Child-Pugh classification A/B/C: 33.3\%/80.0\%/ $100.0 \% ; \mathrm{p}=0.071$ ).

\section{Discussion}

Sarcopenia, a decrease in skeletal muscle mass, has recently been received increased attention. The condition is classified as primary sarcopenia, which is primarily caused by aging, and secondary sarcopenia, which is caused by other events. Secondary sarcopenia is classified into three subcategories: activity-related, disease-related, and nutritionrelated sarcopenia (19). Among these subcategories, sarcopenia is easily induced by complicated processes, such as protein-energy malnutrition, increased basal metabolism, the presence of a portal circulatory shunt (21), as well as elevated serum myostatin levels (which occur secondary to decreased ammonia clearance and inhibit muscle growth) $(22,23)$, and a decreased cognitive function (24), all of which may occur in patients with liver cirrhosis. Sarcopenia, is highly prevalent in patients with liver cirrhosis, with reported rates ranging from $40 \%$ to $70 \%$. This condition significantly affects a patient's ability to perform their activities of daily living (25).

Muscle cramps associated with liver cirrhosis significantly reduce patient QOL (5). While its underlying pathogenesis is unclear, factors such as neurological dysfunction, energy metabolism, electrolyte imbalance, decreased plasma volume, use of loop diuretics (25), vitamin D deficiency (26), ascites, and decompensated cirrhosis (27) are associated with its occurrence. A higher dose of loop diuretics reduces the skeletal muscle mass in patients with liver cirrhosis more rapidly, independent of the severity of liver damage, and is an important risk factor for sarcopenia and decreased survival (25). However, it remains unclear whether sarcopenia is associated with muscle cramps (27). In contrast, Nishiguchi et al. reported that sarcopenia reduces muscle strength and quality to such an extent that normal activities overload the muscles and induce muscle cramps (28).

The results of the current study suggest that the liver function was apparently lower in patients who experienced muscle cramps than in those who did not. Furthermore, the ALBI grade was an objective measure of liver damage because its calculation only required the measurement of the serum Alb and T.Bil levels $(8,9)$. Similarly, the CONUT method (14), which can be calculated using serum Alb, Tcho, and TLC levels, was used to objectively and repeatedly evaluate the patient nutritional status and demonstrated that patients who experienced muscle cramps had a significantly poorer nutritional status in comparison to those who did not. Furthermore, as CT examinations are commonly performed for patients with liver cirrhosis, the evaluation of the skeletal muscle mass is also recommended. The first edition of the criteria for sarcopenia proposed by the Japanese Society of Hepatology in 2016, suggested that skeletal muscle mass should be evaluated using PMI (20). The skeletal muscle mass evaluated by PMI in patients with muscle cramps was significantly decreased. The PMI, ALBI grade, and CONUT method are all objective tools that can be used to predict the risk of developing muscle cramps in daily medical care.

This study revealed that only $59 \%$ of the patients who experienced muscle cramps actually received medical treatment. One possible explanation is that some patients with muscle cramps may not have mentioned the presence of muscle cramps to their doctors due to their lack of knowledge regarding the relationship between liver cirrhosis and these symptoms (27). This study also revealed that a total of $55.7 \%$ of the respondents experienced muscle cramps; accordingly, it is important to educate patients about muscle cramps as a complication of liver cirrhosis and to ask them about muscle cramps in daily practice.

This exploratory study was associated with some limitations. First, this observational study was performed in a single institution with a limited number of patients and a relatively small sample size, which limited the power of the analysis. In particular, a multivariate analysis could not show significant predictors of muscle cramps; thus, it is considered necessary to increase the number of patients and centers in the future. Second, the effect of different etiologies of liver cirrhosis on muscle cramps was not examined. Third, the recent consensus recommendation defined sarcopenia as a demonstrable decline in muscle mass and muscle strength; however, this study only evaluated muscle mass. Fourth, it is difficult to determine the therapeutic effect because no questionnaire was administered before and after the introduction of medications for muscle cramps. Finally, improvements in sarcopenia based on diet, exercise, and other therapeutic interventions should be further considered.

\section{Conclusion}

This survey revealed that more than half of patients with liver cirrhosis experienced muscle cramps and that a substantial number of them did not receive adequate treatment. 
Furthermore, muscle cramps were highly correlated with decreases in the liver function, nutritional status, and skeletal muscle mass.

The authors state that they have no Conflict of Interest (COI).

\section{References}

1. D'Amico G, Garcia-Tsao G, Pagliaro L. Natural history and prognostic indicators of survival in cirrhosis: a systematic review of 118 studies. J Hepatol 44: 217-231, 2006.

2. Konikoff F, Theodor E. Painful muscle cramps. A symptom of liver cirrhosis? J Clin Gastroenterol 8: 669-672, 1986.

3. Kato A, Tanaka H, Kawaguchi $\mathrm{T}$, et al. Nutritional management contributes to improvement in minimal hepatic encephalopathy and quality of life in patients with liver cirrhosis: a preliminary, prospective, open-label study. Hepatol Res 43: 452-458, 2013.

4. Angeli P, Albino G, Carraro P, et al. Cirrhosis and muscle cramps: evidence of a causal relationship. Hepatology 23: 264-273, 1996.

5. Iwasa M, Karino Y, Kawaguchi $T$, et al. Relationship of muscle cramps to quality of life and sleep disturbance in patients with chronic liver diseases: a nationwide study. Liver Int 38: 23092316, 2018.

6. Pugh RN, Murray-Lyon IM, Dawson JL, Pietroni MC, Williams R. Transection of the oesophagus for bleeding oesophageal varices. Br J Surg 60: 646-649, 1973.

7. Hanai T, Shiraki M, Nishimura K, et al. Sarcopenia impairs prognosis of patients with liver cirrhosis. Nutrition 31: 193-199, 2015.

8. Hiraoka A, Kumada T, Michitaka K, et al. Usefulness of albuminbilirubin grade for evaluation of prognosis of 2584 Japanese patients with hepatocellular carcinoma. J Gastroenterol Hepatol 31: 1031-1036, 2016.

9. Hiraoka A, Michitaka K, Kumada T, et al. Validation and potential of albumin-bilirubin grade and prognostication in a nationwide survey of 46,681 hepatocellular carcinoma patients in Japan: the need for a more detailed evaluation of hepatic function. Liver Cancer 6: 325-336, 2017.

10. Johnson PJ, Berhane S, Kagebayashi C, et al. Assessment of liver function in patients with hepatocellular carcinoma: a new evidence-based approach-the ALBI grade. J Clin Oncol 33: 550558, 2015.

11. Hiraoka A, Kumada T, Itobayashi E, et al. Prognostic Scoring System for radiofrequency ablation: Usefulness of Albumin-bilirubin (ALBI)-grade. Kanzo 57: 312-319, 2016 (in Japanese, Abstract in English).

12. Hiraoka A, Kumada T, Kudo M, et al. Hepatic function during repeated TACE procedures and prognosis after introducing sorafenib in patients with unresectable hepatocellular carcinoma: multicenter analysis. Dig Dis 35: 602-610, 2017.

13. Tada $T$, Kumada $T$, Toyoda $H$, et al. Impact of albumin-bilirubin grade on survival in patients with hepatocellular carcinoma who received sorafenib: an analysis using time-dependent receiver operating characteristic. J Gastroenterol Hepatol 34: 1066-1073, 2019.
14. Ignacio de Ulíbarri J, González-Madroño A, de Villar NG, et al. CONUT: a tool for controlling nutritional status. First validation in a hospital population. Nutr Hosp 20: 38-45, 2005.

15. Larramona GL, Lucendo AJ, Tenias JM. Association between nutritional screening via the Controlling Nutritional Status index and bone mineral density in chronic liver disease of various etiologies. Hepatol Res 45: 618-628, 2015.

16. Taniguchi E, Kawaguchi T, Otsuka M, et al. Nutritional assessments for ordinary medical care in patients with chronic liver disease. Hepatol Res 43: 192-199, 2013.

17. Fukushima K, Ueno Y, Kawagishi N, et al. The nutritional index 'CONUT' is useful for predicting long-term prognosis of patients with end-stage liver diseases. Tohoku J Exp Med 224: 215-219, 2011.

18. Rosenberg IH. Sarcopenia: origins and clinical relevance. J Nutr 127: 990S-991S, 1997.

19. Cruz-Jentoft AJ, Baeyens JP, Bauer JM, et al. Sarcopenia: European consensus on definition and diagnosis: report of the European working group on sarcopenia in older people. Age Ageing 39: 412-423, 2010.

20. Nishikawa H, Shiraki M, Hiramatsu A, Moriya K, Hino K, Nishiguchi S. Japan Society of Hepatology guidelines for sarcopenia in liver disease (1st edition): recommendation from the working group for creation of sarcopenia assessment criteria. Hepatol Res 46: 951-963, 2016.

21. Kim G, Kang SH, Kim MY, Baik SK. Prognostic value of sarcopenia in patients with liver cirrhosis: a systematic review and meta-analysis. PLos One 12: e0186990, 2017.

22. McPherron AC, Lawler AM, Lee SJ. Regulation of skeletal muscle mass in mice by a new TGF-beta superfamily member. Nature 387: 83-90, 1997.

23. Nishikawa H, Enomoto H, Ishii A, et al. Elevated serum myostatin level is associated with worse survival in patients with liver cirrhosis. J Cachexia Sarcopenia Muscle 8: 915-925, 2017.

24. Nishikawa H, Enomoto H, Yoh K, et al. Association between sarcopenia and depression in patients with chronic liver diseases. $\mathrm{J}$ Clin Med 8: 634, 2019.

25. Hanai T, Shiraki M, Miwa T, et al. Effect of loop diuretics on skeletal muscle depletion in patients with liver cirrhosis. Hepatol Res 49: 82-95, 2019.

26. Chatrath H, Liangpunsakul S, Ghabril M, Otte J, Chalasani N, Vuppalanchi R. Prevalence and morbidity associated with muscle cramps in patients with cirrhosis. Am J Med 125: 1019-1025, 2012.

27. Sawada Y, Kawaratani H, Kubo T, et al. Effect of furosemide on muscle cramps in patients with liver cirrhosis. J Gastroenterol Hepatol 35: 76-81, 2020.

28. Nishiguchi S. Manual for Treatment of Liver Cirrhosis. Nankodo, Tokyo, 2019: 147-151 (in Japanese).

The Internal Medicine is an Open Access journal distributed under the Creative Commons Attribution-NonCommercial-NoDerivatives 4.0 International License. To view the details of this license, please visit (https://creativecommons.org/licenses/ by-nc-nd/4.0/). 